\title{
Impact of the intellectual environment of professional activity on resting state EEG in older adults
}

\author{
N. Volf ${ }^{1,2 *}$, E. Privodnova ${ }^{1}$ \\ ${ }^{1}$ Scientific Research Institute of Physiology and Basic Medicine, Novosibirsk, Russian Federation \\ ${ }^{2}$ Novosibirsk State University, Novosibirsk, Russian Federation \\ *e-mail:volf@physiol.ru
}

Key words: resting state EEG, old age, gender, mental activity

Motivation and Aim: Although there are clear common principles that can be demonstrated in cognitive aging, enormous variability exists across individuals. A question of great interest is what accounts for this variability. Among other factors inter-individual variability is attributable to involvement in mental activity. The purpose of this study is to outline the changes resting state EEG in aged men and women associated with difference in intellectual environment of their professional activity.

Methods and Algorithms: Elderly (age 63.8+0.5) scientists $(\mathrm{N}=52, \mathrm{SA})$ and people unrelated to professional scientific activity $(\mathrm{N}=63$, NSA) participated in the study. All subjects were employed full time. EEG was recorded from 26 symmetrical regions of the hemispheres in a states with closed and with open eyes. Power spectral density was calculated in delta, theta, alpha 1,2,3, beta 1, 2 and gamma frequency ranges. The bandwidths for the frequency bands were defined using individual alpha peak frequency as the anchor point. The mean power spectral density values were calculated for frontal (Fp1/Fp2, AF3/AF4, F7/F8, F5/F6, F3/F4, F1/F2), central (FC3/FC4, FC1/FC2, C3/C4, C1/C2, CP3/CP4, CP1/CP2), central-temporal (FT7/FT8, FC5/FC6, T7/T8, C5/C6, TP7/TP8, CP5/CP6) and parietal-occipital (P7/P8, P5/P6, P3/P4, P1/P2, PO7/PO8, PO5/ $\mathrm{PO} 6, \mathrm{PO} 3 / \mathrm{PO} 4, \mathrm{O} 1 / \mathrm{O} 2 /$ ) cortical regions separately for the right and the left hemispheres. Results: In condition with closed eyes, ANOVA revealed significant gender $\times$ frequency band $\times$ region $\times$ group $($ SA, NSA) interaction. Testing of interaction demonstrated effects in alpha 2, 3, rhythms power density, characterized by opposite changes of anteroposterior spectral power gradients in SA and NSA groups of men and women. There was no difference between SF and NSA groups in frontal power density. Among women members of ND group had lower posterior power than those of NND group. Conversely, among men, members of ND had greater posterior spectral power in comparison with NND. In eyes open condition, significant asymmetry due to higher power values in right in comparison with left hemisphere in frontal and temporal regions was found in SA group. Asymmetry of power values was not revealed in NSA.

Conclusion: This result of the study confirmed dividing the mentally healthy older population into two subgroups according to levels of intellectual loads during the course of professional activity: significant differences exist in terms of resting state anteroposterior network organization and in hemispheric asymmetry measures.

Acknowledgements: Supported by the RFBR (No. 17-46-540705). 